\title{
Permeability of high-Kn real gas flow in shale and production prediction by pore-scale modeling
}

\author{
Ziyan Wang, Yangyu Guo, Moran Wang ${ }^{\dagger}$ \\ Department of Engineering Mechanics and CNMM, Tsinghua University, Beijing 100084, China
}

\begin{abstract}
Although shale gas has been commercially exploited, the gas transport mechanism in shale is still unclear. Because nanoscale pores are dominant in shale, the Knudsen number of the flow is relatively high so that the conventional Darcy's law fails. What is more, the shale gas in situ is under high pressure and high temperature so that the real gas (or non-ideal gas) effect is significant. Aiming at these two challenges, we did a pore-scale modeling by using lattice Boltzmann method in this work. We developed a pore-field-iteration (PFI) method to bridge up the pore-scale modeling results with the field-scale concerns, such as inflow performance relationship and decline curve analysis. Our results show that the high Knudsen effect leads to a higher gas flow rate, while the real gas effect causes lower gas flow rate. The gas production may be overestimated at early stage due to the real gas effect, while underestimated at late stage because of the high Knudsen number effect. These results may be very helpful for better understanding of gas transport mechanism in shale and for possible process optimization of shale gas developments in future.
\end{abstract}

Keywords: Shale gas; Knudsen number; real gas effect; microporous media; lattice Boltzmann method

\footnotetext{
${ }^{\dagger}$ Corresponding author. Tel.: +86 10627 87498, E-mail: mrwang@ tsinghua.edu.cn
} 


\section{Introduction}

Shale gas is an unconventional gas resource with huge storage. With the gradual declination in conventional gas production, shale gas will play an important role in world energy supply. However, the mechanism of gas flow in shale is not clearly understood yet, as the conventional Darcy's law cannot describe the flow very well [1]. Because of the lacking in fundamental theory and model, key problems such as how to interpret the history production data, how to predict the future production remain unsolved.

In physical view, there are two challenges in modeling the shale gas flow. The first one is the high Knudsen number $(K n)$ effect. As the nanoscale pores are dominated in shale [2; 3], the gas flow in shale has a relative high $K n$ and the permeability in Darcy's law needs to be corrected. Many works have been done focusing on the high Kn effect. Klinkenberg [4] first proposed a correction in which gas permeability increases with $K n$ linearly. After that, Beskok and Karniadakis [5] presented a non-linear correction (termed as Beskok's correction here after for simplicity) for gas flow in straight channel and pipe, which is valid for a wide range of $K n$. Latter, Civan [6] extended Beskok's correction to flow in porous media, based on the use of hydrodynamic radius in defining the $K n$. More recently, the pore-network modeling, in which the complex porous structure is simplified as pore nodes connected by pore bonds, has been used to capture the high $K n$ effect $[7 ; 8]$.

The second challenge is the real gas effect (or non-ideal gas effect). In most of the reservoirs, shale gas is a kind of supercritical fluid which cannot be treated as ideal gas. In 1966, Hussainy et al. [9] proposed the famous pseudo-pressure method to linearize the flow equations of real gas. No further works have been done regarding this problem until in recent years. Wang and $\mathrm{Li}[10 ; 11 ; 12]$ developed a Monte Carlo method based on the Enskog theory to simulate high $\mathrm{Kn}$ real gas flows, 
which is though very expensive for porous media flow and its application is merely limited to channel flow. Michel et al. [13] combined the Beskok's correction with the pseudo-pressure method, to consider both the Kn effect and real gas effect. Wang and Marongiu-Porcu [14] recently used another correction to describe high $K n$ effect and coupled the real gas effect, formation compaction and gas desorption in their numerical simulations.

However, most of these models are in Darcy scale. They are accurate for regular porous media, but problematic for complex, heterogeneous and hierarchical structures like shale because of ignored effects of geometry details. With the developments of lattice Boltzmann method (LBM) which holds the advantage in tackling complex boundaries[15], it becomes possible to directly simulate gas flows in complex structures, i.e. by pore-scale modeling [16]. Pore-scale modeling is capable of capturing all the geometry details, therefore it is highly accurate for flow behavior in complex structure.

One challenge for pore-scale modeling of shale gas is the multiscale issue. The target is to understand and predict the production at field scale. However based on the computational cost, the pore-scale simulation is usually limited to very small volume[17]. An efficient upscaling method is desperately needed to bridge the microscale simulation results and the macroscale prediction.

In this work, we firstly simulate the high $K n$ real gas flow at pore scale by using LBM. Several benchmarks are made to validate our numerical method. Then a multiple-scale integration method is proposed to upscale the pore-scale simulations to field-scale problems. To predict the production of shale gas, field-scale problems such as the inflow performance relationship (IPR) and decline curve analysis (DCA) are studied.

\section{Numerical method}

\subsection{High Knudsen number effect}


Knudsen number is defined as [18],

$$
K n=\frac{\lambda}{l},
$$

where $\lambda$ and $l$ are the mean free path of gas molecules and the characteristic length of the structure, respectively. The real gas mean free path model proposed by Bird [19] is adopted here: $\lambda=\frac{\mu}{\rho} \sqrt{\frac{\pi M}{2 R T}}$, where $\mu$ is the dynamic viscosity of gas; $\rho$ is the gas density and $M$ is the molar mass. For the characteristic length, the hydrodynamic tube radius of the well-known Kozeny-Carman model [20] is applied: $r=2 V_{p} / S_{P}$, where $V_{p}$ is the total pore volume, $S_{p}$ is the interface area between pore and solid matrix. For a straight channel, the channel height is taken as the characteristic length.

To simulate high $K n$ flow, lattice Boltzmann method (LBM) is used because of two aspects: (1) it has solid physical foundation since the lattice Boltzmann equation is derived directly from Boltzmann equation $[15 ; 21]$, which is the fundamental transport law available for high $K n$ flow; (2) LBM has a high efficiency in dealing with complex boundaries of porous structure[22; 23]. LBM describes the evolution of the discrete distribution function, which can be written as

$$
f_{i}\left(\mathbf{x}+\mathbf{c}_{i} \Delta t, t+\Delta t\right)-f_{i}(\mathbf{x}, t)=\Omega_{i}(f), \quad i=0,1, \cdots, N-1
$$

where $f_{i}(\mathbf{x}, t)$ is the discrete distribution function, $\Omega_{i}(f)$ is the discrete collision operator, $N$ is the number of the discrete velocities. For numerical accuracy, the 3-dimension 19-speed model (D3Q19) is used in this work[24]. There are usually two models for the collision operator: single relaxation time model (SRT) [25; 26] and multiple relaxation time model (MRT) [27; 28]. For the numerical stability concerns of SRT model for variable-viscosity flows in porous media, the MRT model is applied in the present work $[29 ; 30]$ :

$$
\Omega_{i}=-\sum_{j}\left(\mathbf{M}^{-1} \mathbf{S M}\right)_{i j}\left(f_{j}-f_{j}^{e q}\right)
$$


The features of high $K n$ flow mainly include two aspects: (1) a gas slip adjacent to the wall; (2) a lower effective viscosity of gas. In LBM, the wall gas slip can be captured by the discrete diffuse boundary condition (or the discrete Maxwellian boundary condition) [31], and the gas viscosity is described by the Bosanquet-type effective viscosity model,

$$
\mu_{e}=\frac{\mu}{1+a K n}
$$

with the empirical parameter $a=2.2$, suggested by Beskok and Karniadakis [5].

To validate our numerical method, the channel flow is simulated as a benchmark. The grid is $200 \times 10$ and the fluid density $\rho$ is doubled from $1 \mathrm{~kg} / \mathrm{m}^{3}$ to $256 \mathrm{~kg} / \mathrm{m}^{3}$ successively. Other parameters are listed in Table 1. The fluid is treated as ideal gas. Fig. 1(a) compares the cross-section velocity profile obtained by present LBM simulation with Beskok's model [5] and Fig. 1(b) compares the normalized mass flow rate $Q=\frac{\rho u_{\text {avg }}}{(-d p / d x) H} \sqrt{\frac{2 R T}{M}}$ in the present work with Cercignani's theoretical solution [32]. The agreement between our numerical simulations and the theoretical models is very good. Thus the present numerical framework for high $K n$ flow is reliable for further exploration.

Table 1 Parameters for high $K n$ flow simulation

\begin{tabular}{|l|c|}
\hline Temperature $T(\mathrm{~K})$ & 373 \\
\hline Molar mass $M(\mathrm{~kg} / \mathrm{mol})$ & 0.016 \\
\hline Pressure gradient $\nabla p(\mathrm{MPa} / \mathrm{m})$ & 1 \\
\hline Channel height $H(\mathrm{~nm})$ & 10 \\
\hline Dynamic viscosity $\mu\left(\mathrm{kg} \cdot \mathrm{m}^{-1} \cdot \mathrm{s}^{-1}\right)$ & $1 \times 10^{-5}$ \\
\hline
\end{tabular}




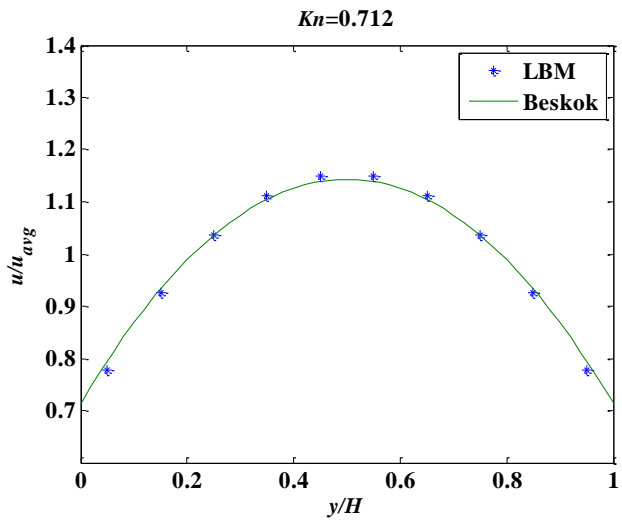

(a)

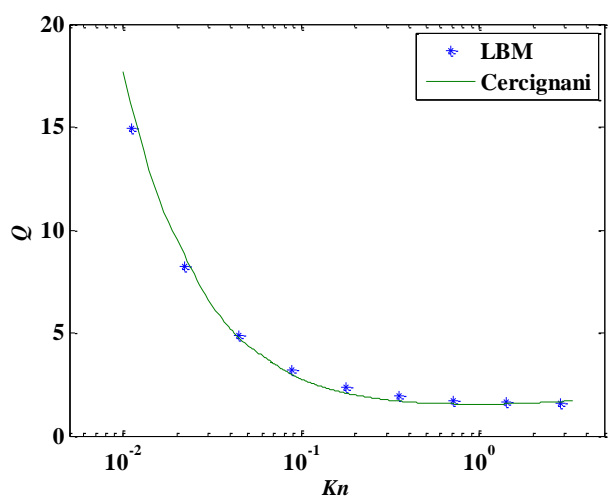

(b)

Fig. 1 Comparison of LBM simulation and theoretical modeling of high $K n$ flow in channel: (a) velocity profile in the cross section of the channel at $K n=0.712$, (b) normalized mass flow rate versus $K n$.

\subsection{Real gas effect}

In the present work, the shale gas is considered as pure methane for simplicity, since the mole fraction of methane is usually more than $90 \%$ in shale gas. Real gas effect also involves two aspects: (1) the equation of state (EOS) of real gas, (2) variable properties of real gas. For the first aspect, Redlich-Kwong (RK) EOS is able to give a simple but accurate description for gas at high pressure and temperature [33], which is written as

$$
p=\frac{R T}{V_{m}-b}-\frac{a}{\sqrt{T} V_{m}\left(V_{m}+b\right)},
$$

where $p$ is the gas pressure; $T$ is the absolute temperature; $R$ is the gas constant; $V_{m}$ is the molar volume; $a$ and $b$ are constants. The RK equation works well as is shown in Fig. 2 (a) through a comparison with NIST standard data [34]. For the second aspect, the pressure-dependent dynamic viscosity is considered, which is usually a constant at a certain temperature for ideal gas. The NIST dynamic viscosity data for methane [35] is adopted in the present work as is shown in Fig. 2 (b). 


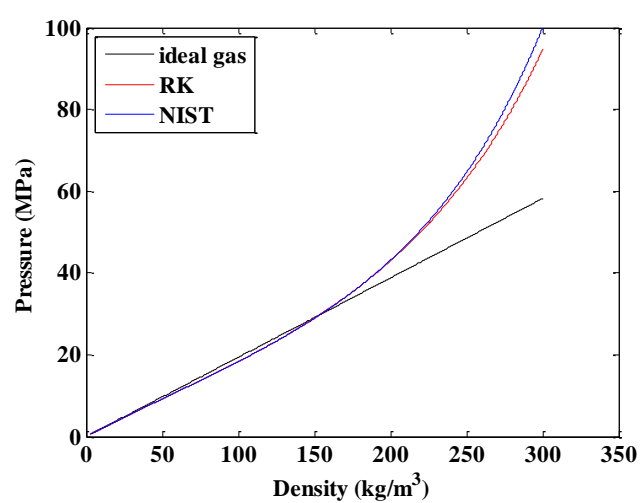

(a)

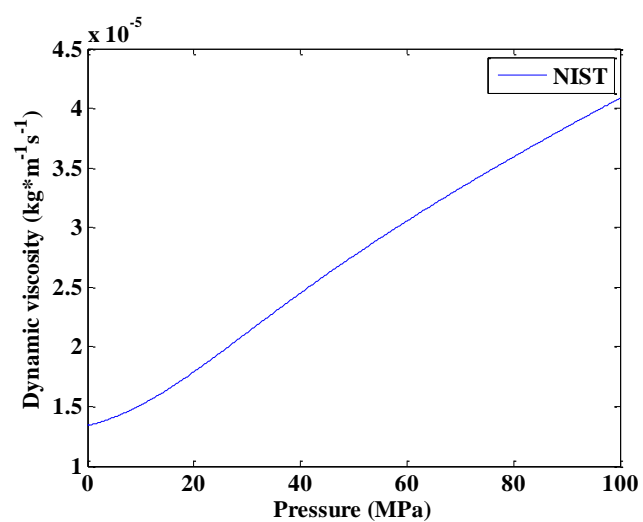

(b)

Fig. 2 Real gas effect of shale gas (methane): (a) Equation of state (EOS) of methane. (b) Dynamic viscosity versus pressure. The temperature is $373 \mathrm{~K}$.

To accurately capture the real gas effect, our simulation should be reliable for variable properties of gas, which means the numerical results must be independent with relaxation time in LBM. Therefore, the non-slip flow in body centered cube (BCC) array of spheres is simulated as a validation. The parameters are given in Table 2 and the results are presented in Fig. 3 (similar results reported in Ref. [30]). The MRT model for collision operator results in much less numerical error than SRT under the same grid resolution, thus is more reliable and effective for porous flow simulation with variable gas properties. This explains why the simple and widely used SRT model is abandoned in the present work.

Table 2 Parameters for flow in BCC structure

\begin{tabular}{|l|c|}
\hline Side length $(\mathrm{nm})$ & 320 \\
\hline Porosity & 0.582 \\
\hline Grid number of one side, $N$ & $32,64,96$ \\
\hline Relaxation time & $0.6 \sim 2$ \\
\hline Theoretical permeability $(\mathrm{mD})$ & 0.2896 \\
\hline
\end{tabular}




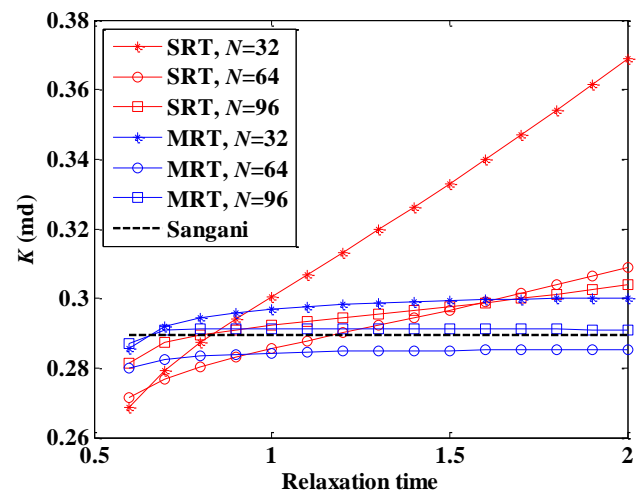

Fig. 3 Comparison of LBM results with SRT and MRT models for the collision operator: the permeability of BCC structure versus relaxation time. The dashed line is the theoretical result from Sangani and Acrivos [36].

For an intuitive illustration of the importance of real gas effect, Fig. 4 (a) compares the results of $K n$ computed based on the ideal gas model and real gas model respectively. Through a simulation of high $K n$ flow, Fig. 4 (b) presents the difference of permeability ratio between ideal gas and real gas flow. The real gas effect is shown to be remarkable at typical shale gas pressure and temperature.

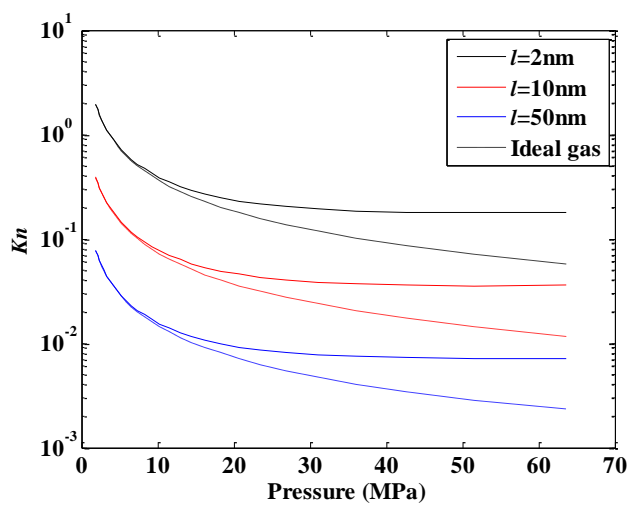

(a)

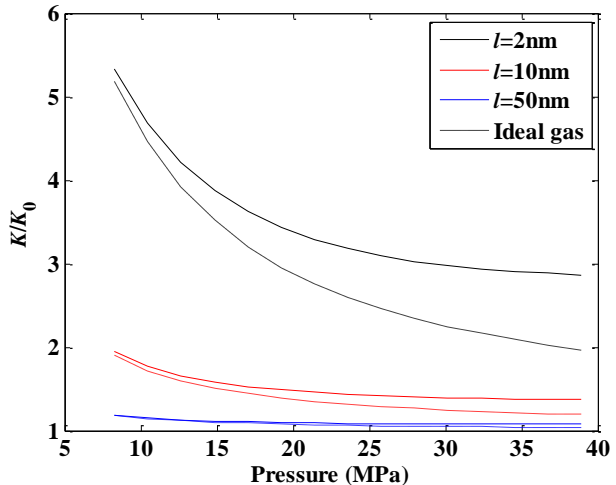

(b)

Fig. 4 Difference between ideal gas and real gas: (a) $K n$ versus pressure at different characteristic lengths. (b) Permeability ratio versus pressure at different characteristic lengths based on channel flow simulation. The solid lines represent the real gas results, while the dashed lines represent the ideal gas results. The temperature is $373 \mathrm{~K}$.

\subsection{Upscaling method}


To link the pore-scale simulation results and field-scale problem, here we propose an upscaling method: pore-field-iteration (PFI). In PFI, the pore-scale simulation is the input and the field-scale problem is the output. The pore-scale properties, including the microstructure geometry effect, will propagate to the field scale through iterations. To illustrate PFI method, we firstly consider the steady state isothermal flow along a long straight porous media. The pressure at left side is the average reservoir pressure $p_{r}$ and at right side is the bottom hole pressure $p_{w}$ (Fig. 5). It can be interpreted that there is a hydraulic fracture at left boundary which has good connection to the wellbore, so its pressure equals the bottom hole pressure. The pressure distribution and mass flow rate at steady state is obtained through an iterative solution in the PFI process described as below:

(1) Uniformly divide the porous media into several zones. In the center of each zone, take a representative volume elementary (REV) bounded within the black square shown in Fig. 5. (The REV is enlarged for a clear illustration.)

(2) Give an initial pressure distribution along the porous media.

(3) Determine the center pressure and pressure difference between the inlet and outlet of each REV.

(4) Simulate the flow in each REV under the given pressure difference and obtain the mass flow rate.

(5) Update the pressure distribution based on the mass flow rate. Mathematical details of the updating method will be introduced soon.

(6) Repeat (3) (5) until the pressure distribution is converged. Then output the results. 


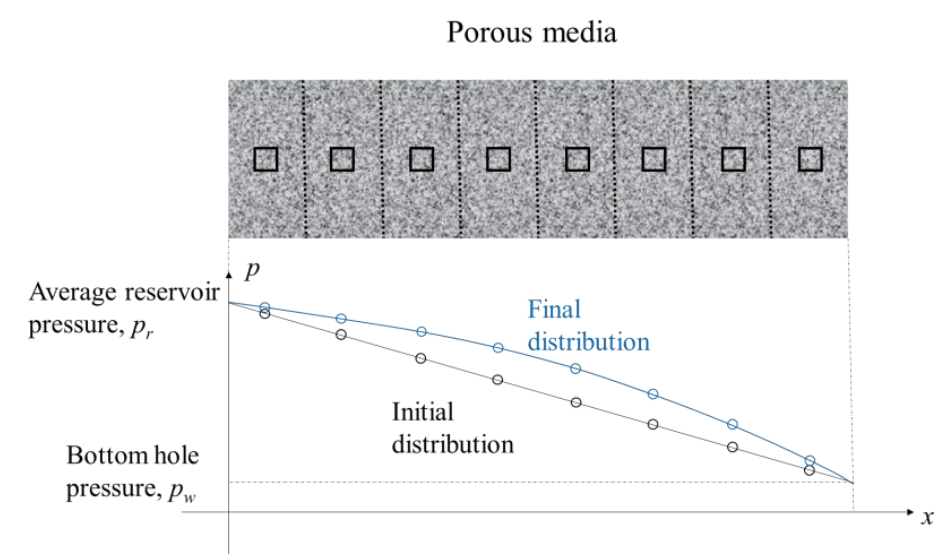

Fig. 5 Schematic of the pore-field-iteration (PFI) upscaling method: the pressure distribution along the field-scale porous media. A linear initial pressure distribution is shown by black line and after iteration the distribution will be non-linear as shown in blue line.

The basic idea of the updating method is to approach a consistent 'target variable' for all REVs. For steady state flow without internal fluid source or sink, the target variable is the mass flow rate restricted from the mass balance law. Thus once the mass flow rate is higher than the average value, it is essential to reduce pressure difference between the inlet and outlet of the corresponding REV to lower the flow rate down. For the $i^{\text {th }} \mathrm{REV}$, an effective updating method is therefore proposed here as:

$$
\begin{aligned}
& \Delta p_{i}^{\text {new }}=\Delta p_{i}-\frac{1}{N_{R E V}}\left(p_{r}-p_{w}\right)\left(q_{i}-\bar{q}\right) / \bar{q}, \\
& p_{i}^{\text {new }}=p_{r}-\sum_{j=1}^{i} \Delta p_{j}^{\text {new }}+\frac{1}{2} \Delta p_{i}^{\text {new }},
\end{aligned}
$$

where $\Delta p_{i}$ is the pressure difference between inlet and outlet; $p_{i}$ is center pressure; $N_{R E V}$ is the total number of REV; $q_{i}$ is the mass flow rate and $\bar{q}=\frac{1}{N_{R E V}} \sum_{i} q_{i}$ is the average mass flow rate. EQ.(6) is constructed to lower down iteration error. Any updating methods which can adjust the pressure distribution to make the mass flow rate uniform can be applied in PFI. After the updating, the flow will be simulated in each REV according to the new pressure and pressure difference.

The real process of gas exploitation is an unsteady state one. Therefore a pseudo-steady state model is much more useful for gas production prediction, especially when the drainage area is 
bounded or there are several wells in the field [37]. The model assumes the gas pressure drops uniformly in every point of the gas field, so the target variable is the gas pressure drop rate. The process of PFI for pseudo-steady state flow is the same as that for steady state flow, except the updating method becomes slightly different:

$$
\begin{aligned}
& \Delta p_{i}^{\text {new }}=\Delta p_{i}-\frac{1}{N_{R E V}}\left(p_{r}-p_{w}\right)\left(q_{i}-f_{i}\right) / \bar{q}, \\
& p_{i}^{\text {new }}=p_{r}-\sum_{j=1}^{i} \Delta p_{j}^{\text {new }}+\frac{1}{2} \Delta p_{i}^{\text {new }},
\end{aligned}
$$

where $f_{i}$ is a new parameter determined by

$$
f_{1}=\frac{1}{2} C \frac{d \rho}{d p}_{p=p_{r}}, f_{i+1}-f_{i}=C{\frac{d p}{d \rho_{p=p_{i}}}}_{i}, \sum_{i} f_{i}=\sum_{i} q_{i},
$$

where $C$ is a constant and can be eliminated.

To verify the PFI method, steady state flow in a long straight channel is simulated as a benchmark. The real gas is methane with its properties described in the subsection 2.2 , and ideal gas model is also applied as comparison. Simulation parameters are listed in Table 3. As is shown in Fig. 6 , the present numerical results agree well with analytical solutions, which are derived in appendix A. The validation of PFI for pseudo-steady state flow will be proved in the next section.

Table 3 Parameters for field-scale channel flow

\begin{tabular}{|l|c|}
\hline Temperature $T(\mathrm{~K})$ & 373 \\
\hline Average reservoir pressure $p_{r}(\mathrm{MPa})$ & 40 \\
\hline Bottom hole pressure $p_{w}(\mathrm{MPa})$ & 5 \\
\hline Field length $L(\mathrm{~m})$ & 15 \\
\hline Channel height $H(\mathrm{~nm})$ & 10 \\
\hline REV number $N_{R E V}$ & 16 \\
\hline
\end{tabular}




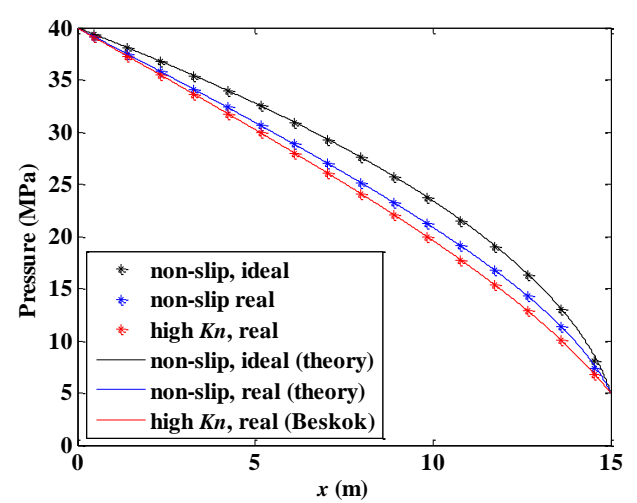

(a)

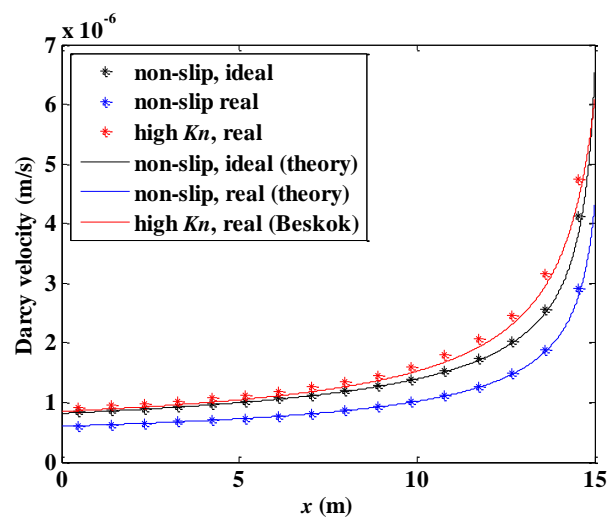

(b)

Fig. 6 Comparison of present simulation to theoretical results of steady state channel flow at field scale: distribution of (a) pressure, (b) Darcy velocity along the long straight channel. Solid lines are theoretical results and points are simulation results.

The significance of this PFI upscaling method is explicit since it provides an avenue to resolve the field-scale problem based on pore-scale modeling. It thus can be also easily applied in many other problems like gas diffusion and electrokinetic flow in field-scale porous media. The PFI method in present work is limited in one-dimensional straight flow, but will be generalized to axial or spherical flows in future work.

\section{Results and discussion}

\subsection{Inflow performance relationship}

The inflow performance relationship (IPR) describes the relation between the gas production and the bottom hole pressure $p_{w}$, under a constant average reservoir pressure $p_{r}$. IPR is of vital significance in analyzing the gas storage, predicting the gas production and optimizing the well arrangement.

In this section, a complex reconstructed porous media generated by QSGS method [38; 39] as a representation of real shale (Fig. 7) is used for numerical simulation and analysis. For an economic computation time without losing physical interpretation, the porosity is set as 0.492 , which is a little 
larger than that of the real shale. The seed density used in the QSGS method is 0.01 , and the generated porous structure is isotropic. More geometry details of shale will be considered in future, as a generalization of the present preliminary study.

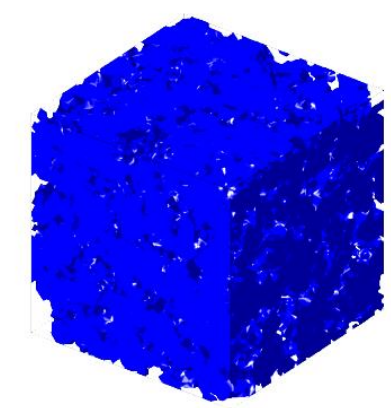

Fig. 7 Reconstructed porous media of shale generated by QSGS method[38]

Firstly, the IPR of steady state flow is considered. In non-slip ideal gas flow, the permeability and viscosity are constant, with the theoretical expression of IPR derived as (see appendix A for details):

$$
q=q_{A O F}\left(1-\frac{p_{w}^{2}}{p_{r}^{2}}\right)
$$

where $q$ is the gas production (mass flow rate at well) and $q_{A O F}$ is the absolute open flow (gas production at vanishing bottom hole pressure). Based on the PFI method, non-slip ideal gas flow through the generated porous structure is simulated. The numerical result displays good agreement with theoretical solution as is shown in Fig. 8. Then, the high $K n$ effect and/or real gas effect are included into the simulation. Fig. 9 shows a comparison of the numerical results under different conditions. The parameters are listed in Table 4.

Table 4 Parameters for IPR of flow in reconstructed porous media of shale

\begin{tabular}{|l|c|}
\hline Temperature $T(\mathrm{~K})$ & 373 \\
\hline Average reservoir pressure $p_{r}(\mathrm{MPa})$ & 40 \\
\hline Bottom hole pressure $p_{w}(\mathrm{MPa})$ & 5 \\
\hline Field length $L(\mathrm{~m})$ & 15 \\
\hline
\end{tabular}




\begin{tabular}{|l|c|}
\hline REV side length (nm) & 100 \\
\hline Grid number of one side, $N$ & 100 \\
\hline REV number, $N_{R E V}$ & 10 \\
\hline
\end{tabular}

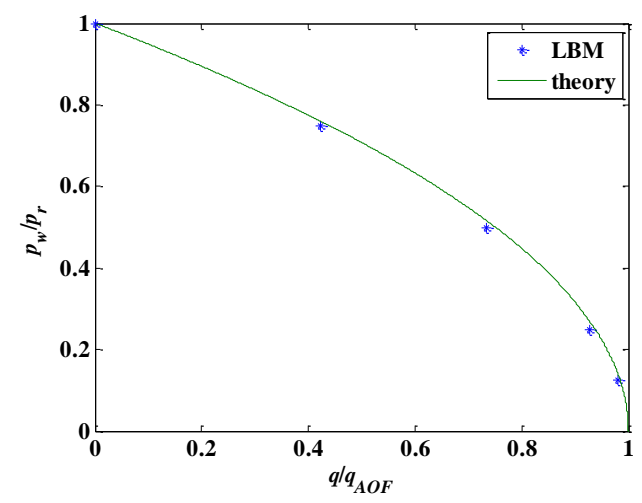

Fig. 8 Comparison of the simulation to theoretical result for the dimensionless IPR curve of non-slip ideal gas flow. The flow is at steady state in reconstructed porous media of shale.

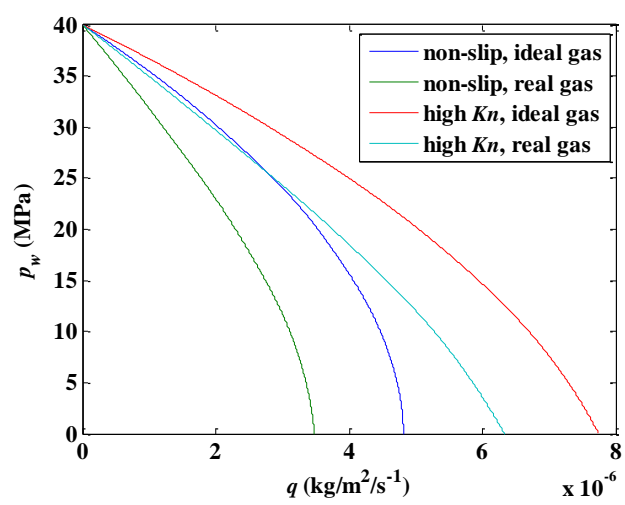

(a)

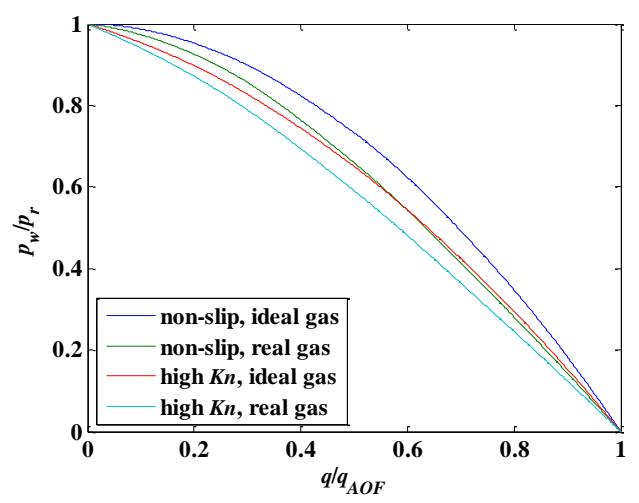

(b)

Fig. 9 IPR curves with high $K n$ effect and/or real gas effect. (a) dimensional results, (b) dimensionless results. The flow is at steady state in reconstructed porous media of shale.

The IPR curve for pseudo-steady state flow is then studied. The theoretical solution for pseudo-steady state flow proposed by Fetkovich [40] is used as a comparison for non-slip ideal gas flow. As is shown in Fig. 10, the numerical result by LBM-based PFI method agrees well with the theoretical solution. Coincidently, the relation presented by Fetkovich is the same with Eq.(9), so Fig. 8 and Fig. 10 look the same. Fig. 11 shows the numerical results with an inclusion of high Kn effect 
and/or real gas effect. All parameters remain the same as those in steady state simulation (Table 4).

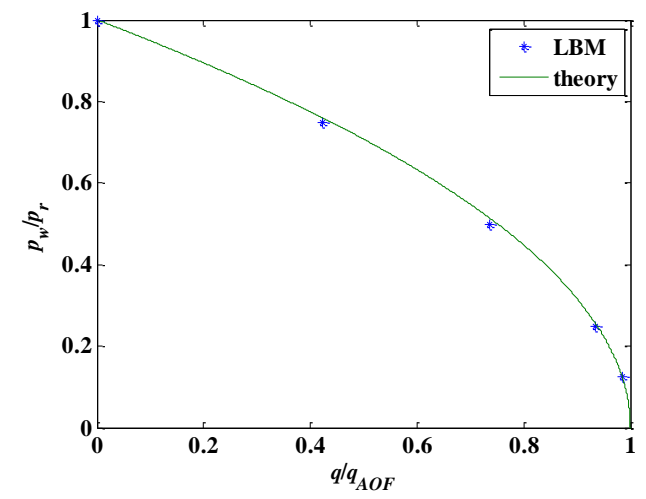

Fig. 10 Comparison of the simulation to theoretical result for the dimensionless IPR curve of non-slip ideal gas flow. The flow is at pseudo-steady state in reconstructed porous media of shale.

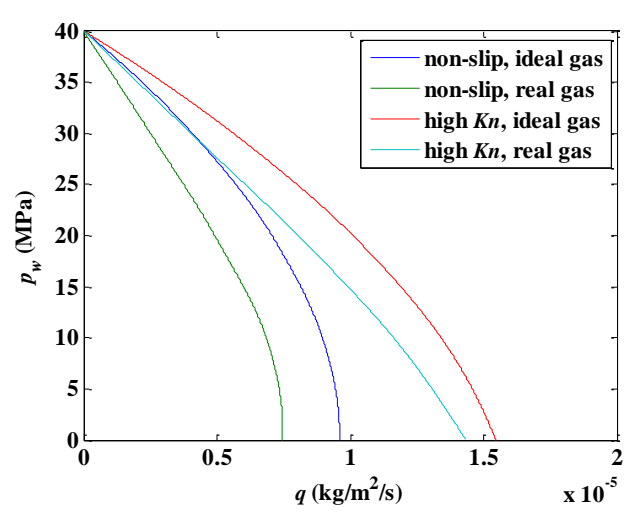

(a)

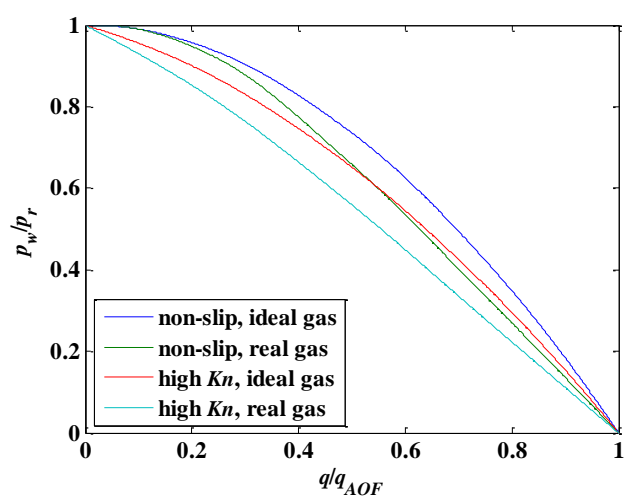

(b)

Fig. 11 IPR curves with high $K n$ effect and/or real gas effect. (a) dimensional results, (b) dimensionless results. The flow is at pseudo-steady state in reconstructed porous media of shale.

For both steady and pseudo-steady state flow through the shale porous structure, the mass flow rate is elevated in high $K n$ flow under the same bottom hole pressure and average reservoir pressure. This is because the permeability with high $K n$ effect is larger attributed to the gas slip adjacent to the solid wall. In contrast, the real gas effect makes the mass flow rate lower, which is mainly caused by the larger viscosity of real gas at high pressure.

It should be noted that the analysis for IPR is not intended to be directly used in shale gas engineering because the transient flow dominate at the beginning of exploitation. However, the analysis provides some semi-quantitative results to evaluate the high $\mathrm{Kn}$ and real gas effect in gas 
production. It is also the fundamental of the decline curve analysis in next subsection.

\subsection{Decline curve analysis}

Decline curve analysis (DCA) is a widely concerned issue in natural gas engineering. In this section, the gas flow is assumed in pseudo-steady state. At the initial state, the average reservoir pressure $p_{r}$ is $40 \mathrm{MPa}$ and the bottom hole pressure $p_{w}$ is $30 \mathrm{MPa}$. As the flow proceeds, the pressure uniformly drops and at the final state, the bottom hole pressure is reduced to 10MPa. The flow is simulated when bottom hole pressure drops to $30,25,20,15,10 \mathrm{MPa}$, with the pressure change rate $d p / d t$ and mass flow rate at bottom hole $q$ computed. Through a spline interpolation, the functions $d p / d t\left(p_{w}\right)$ and $q\left(p_{w}\right)$ are determined from the discrete simulation results. Thus the time $(t)$ is inversely integrated as:

$$
t\left(p_{w}\right)=\int_{p_{w}^{\text {initial }}}^{p_{w}} \frac{1}{\frac{d p}{d t}\left(p_{w}\right)} d p_{w} .
$$

Therefore, quantitative relations are established between bottom hole pressure, gas production and time.

To validate the present numerical method for DCA, the channel flow is simulated firstly. The parameters are listed in Table 5. The decline curves in terms of for bottom hole pressure and gas production are given in Fig. 12(a) and Fig. 12(b) respectively. The numerical result for ideal gas non-slip flow agrees quite well with the corresponding theoretical result, thus verifying the present approach. It is also seen that the decline curves for non-slip ideal gas flow and high $K n$ real gas flow are intersected, which means that the gas production will be overestimated at the early stage and underestimated at the late stage when neglecting the high $K n$ effect and real gas effect. At the early stage, the real gas effect is dominated since the gas pressure is high, which results in larger gas 
viscosity and thus lower gas production. In contrast, the pressure becomes low at the late stage, leading to the dominance of high $K n$ effect, which makes the gas production higher.

Table 5 Parameters for DCA of flow in channel

\begin{tabular}{|l|c|}
\hline Temperature $T(\mathrm{~K})$ & 373 \\
\hline Average reservoir pressure $p_{r}(\mathrm{MPa})$ & $40,35,30,25,20$ \\
\hline Bottom hole pressure $p_{w}(\mathrm{MPa})$ & $30,25,20,15,10$ \\
\hline Field length $L(\mathrm{~m})$ & 15 \\
\hline Channel height $(\mathrm{nm})$ & 10 \\
\hline Grid & $200 \times 10$ \\
\hline REV number, $N_{R E V}$ & 10 \\
\hline
\end{tabular}

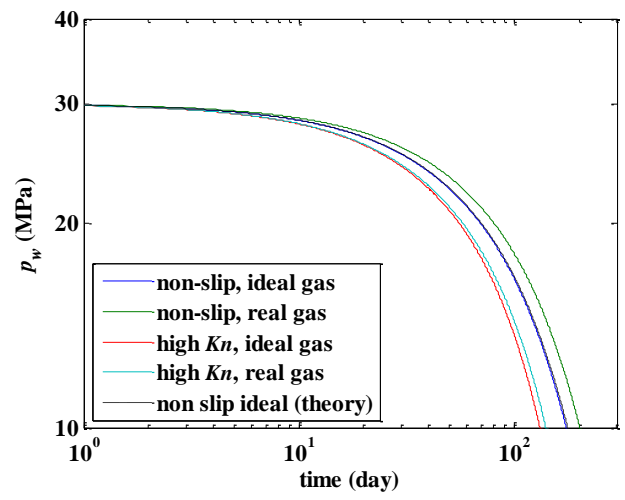

(a)

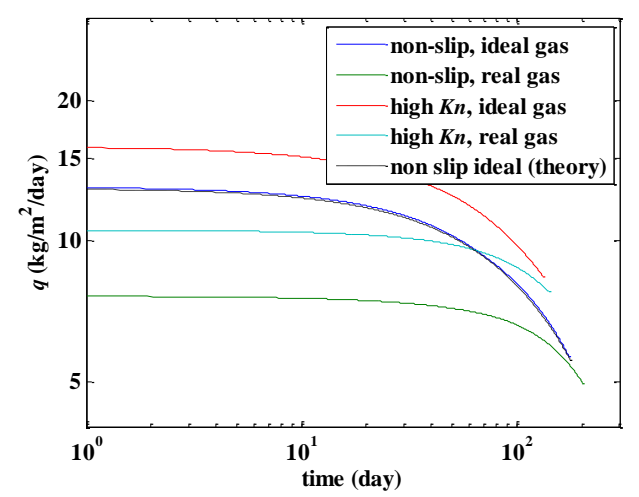

(b)

Fig. 12 Decline curves of (a) bottom hole pressure and (b) gas production for pseudo-steady state flow in channel. Solid lines are numerical results and dashed line is the theoretical result, which is obtained by integrating Eq.(10) analytically for non-slip ideal gas flow.

The flow is then simulated and analyzed in reconstructed porous structure of shale with the same structure parameters in subsection 3.1, and other parameters are given in Table 6. The numerical results are presented in Fig. 13. At early stage, the predicted production is roughly the same for non-slip ideal gas flow and high $K n$ real gas flow. However, an appreciable underestimation of production is observed in non-slip ideal gas model at late stage. This is because at early stage, the high 
$K n$ effect and real gas effect are counteracted, but as the pressure drops down, high $K n$ effect becomes more significant whereas real gas becomes weaker, which gives rise to higher gas production at late stage.

Table 6 Parameters for DCA of flow in reconstructed porous media of shale

\begin{tabular}{|l|c|}
\hline Temperature $T(\mathrm{~K})$ & 373 \\
\hline Average reservoir pressure $p_{r}(\mathrm{MPa})$ & $40,35,30,25,20$ \\
\hline Bottom hole pressure $p_{w}(\mathrm{MPa})$ & $30,25,20,15,10$ \\
\hline Field length $L(\mathrm{~m})$ & 15 \\
\hline REV side length $(\mathrm{nm})$ & 100 \\
\hline Grid number of one side, $N$ & 100 \\
\hline REV number, $N_{R E V}$ & 10 \\
\hline
\end{tabular}

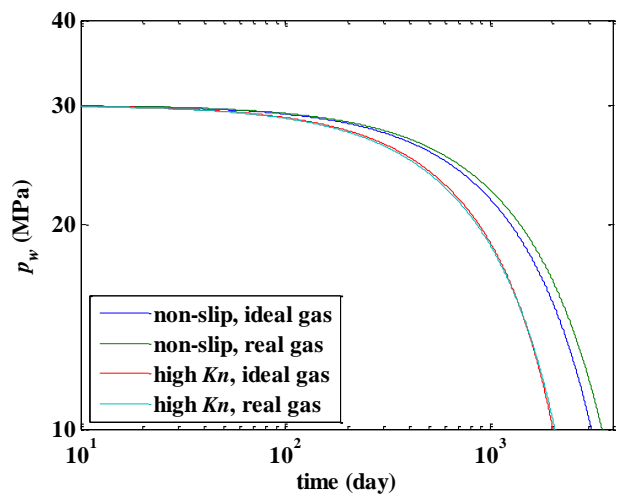

(a)

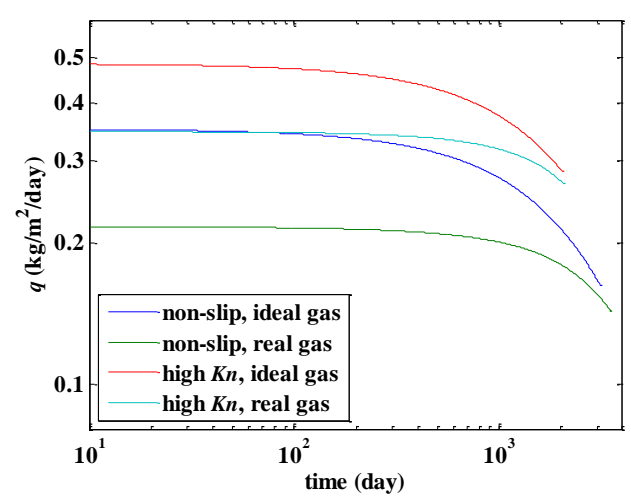

(b)

Fig. 13 Decline curves of (a) bottom hole pressure and (b) gas production for pseudo-steady state flow in in reconstructed porous media of shale.

Moreover, we compare the simulation result of high $K n$ real gas flow in shale with the Arps hyperbolic relation [41]:

$$
q(t)=\frac{q_{i}}{\left(1+b D_{i} t\right)^{1 / b}} .
$$

Since the pseudo-steady state model is not sufficiently accurate at the beginning of the exploitation 
due to the non-uniform pressure change, only the numerical result at late stage is used for a comparison, shown in Fig. 14. The fitting parameters in Eq.(11) are: $q_{i}=0.353 \mathrm{~kg} / \mathrm{m}^{2} /$ day, $D_{i}=1.166 \times 10^{-4}$ day $^{-1}, b=2.319 \times 10^{-4}$. The present work provides a credible semi-quantitative prediction of the production process of shale gas, but there is still much difference between our modeling and realistic situation. Future investigation is necessary of the following aspects: the heterogeneity of structure, the matrix deformation and gas desorption.

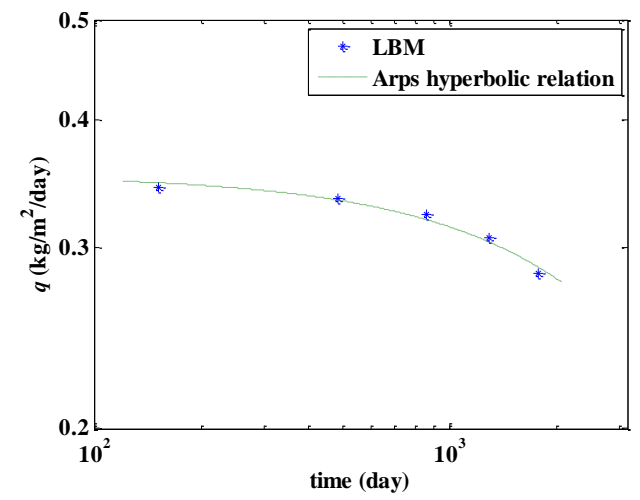

Fig. 14 Comparison of the high $K n$ real gas flow simulation to Arps hyperbolic relation for the production decline curve. The flow is in reconstructed porous media of shale.

\section{Conclusions}

The high-Kn real gas flow in shale is investigated through a two-step strategy: firstly, the flow is simulated at pore scale by LBM; the PFI upscaling method is then proposed to upscale the pore-scale numerical results for solving field-scale problems. Flows in channel and BCC structure are used to validate our pore-scale numerical method in accurately capturing both the high $K n$ effect and real gas effect. Then, the PFI method is verified through a simulation of field-scale channel flow, whose result is in good agreement with theoretical solution. Thus two widely concerned field-scale problems, i.e. IPR and DCA, are resolved in the present new numerical frame. In the study of IPR, it is uncovered that high $K n$ effect leads to a larger mass flow rate while real gas effect leads to a lower flow rate under the same pressure conditions. In DCA, the real gas effect is found to be dominant at the early 
stage while the high $K n$ effect becomes dominant at late stage, which respectively results in an overestimation and an underestimation of the gas production in conventional non-slip ideal gas flow model. The production prediction is also compared with the classical Arps hyperbolic relation and a good match is achieved. Future explorations are still needed including more realistic effects in shale such as the heterogeneous structure, gas desorption and matrix deformation.

\section{Appendix A}

The theoretical result for steady-state isothermal flow is derived. With high $K n$ effect, the mass

flowrate is:

$$
q=\rho u_{D}=-\rho \frac{f(K n) K_{0}}{\mu(p)} \frac{d p}{d x}
$$

where $u_{D}$ is the Darcy velocity, $K_{0}$ the intrinsic permeability and $f$ the gas permeability correction

factor of $K n$ effect. Substitution of the real gas EOS, $p=p(\rho)$, into EQ.(A.1) leads to

$$
q=-\rho \frac{f(K n) K_{0}}{\mu(\rho)} p^{\prime}(\rho) \frac{d \rho}{d x}
$$

For a certain structure, $K n$ is only a function of $\rho$, so EQ.(A.2) can be solved by direct integration:

$$
\begin{gathered}
\int d x=\int-\frac{1}{q} \rho \frac{f(K n) K_{0}}{\mu(\rho)} p^{\prime}(\rho) d \rho, \\
q=\int_{\rho_{r}}^{\rho_{w}}-\frac{1}{L} \rho \frac{f(K n) K_{0}}{\mu(\rho)} p^{\prime}(\rho) d \rho,
\end{gathered}
$$

where $L$ is the field length, $\rho_{w}$ and $\rho_{r}$ are density at bottom hole and reservoir, respectively. The

relation between $\mathrm{x}$ and $\rho$ is built as:

$$
x=\int_{\rho_{r}}^{\rho}-\frac{1}{q} \rho \frac{f(K n) K_{0}}{\mu(\rho)} p^{\prime}(\rho) d \rho .
$$


After determination of density distribution along the flow, the pressure and Darcy velocity distribution can be easily derived.

For channel flow, there is a theoretical value for $K_{0}$,

$$
K_{0}=\frac{H^{2}}{12}
$$

and by applying Beskok's correction,

$$
f(K n)=\left(1+\frac{6 K n}{1+K n}\right)(1+2.2 K n),
$$

the flow can be solved explicitly, as shown in Fig. 6 .

For flow in complex structures, there is no theoretical value of for $K_{0}$ and $f(K n)$ is affected by the geometry details. However, the dimensionless IPR can still be determined for non-slip ideal gas flows. Under that conditions, EQ.(A.4) is simplified as

$$
q=\int_{\rho_{r}}^{\rho_{w}}-\frac{1}{L} \rho \frac{K_{0}}{\mu} \frac{R T}{M} d \rho=\frac{K_{0} M}{2 L \mu R T}\left(p_{r}-p_{w}\right)^{2}
$$

When $p_{w}=0$, The flowrate is the absolute open flowrate:

$$
q_{A O F}=\frac{K_{0} M}{2 L \mu R T} p_{r}^{2}
$$

Therefore we get:

$$
\frac{q}{q_{A O F}}=1-\frac{p_{w}^{2}}{p_{r}^{2}},
$$

which is corresponding with EQ.(9).

Acknowledgements: This work is financially supported by the NSF grant of China (No. 51176089, U1562217), National Science and Technology Major Project on Oil and Gas (No.2016ZX05013001), the PetroChina Innovition Foundation and the Tsinghua University Initiative Scientific Research Program (No. 2014z22074). 


\section{References}

[1] F. Javadpour, D. Fisher, and M. Unsworth, Nanoscale gas flow in shale gas Sediments. Journal of Canadian Petroleum Technology 46 (2007) 55-61.

[2] R.G. Loucks, R.M. Reed, S.C. Ruppel, and D.M. Jarvie, Morphology, genesis, and distribution of nanometer-scale pores in siliceous mudstones of the Mississippian Barnett shale. Journal of Sedimentary Research 79 (2009) 848-861.

[3] G.R. Chalmers, R.M. Bustin, and I.M. Power, Characterization of gas shale pore systems by porosimetry, pycnometry, surface area, and field emission scanning electron microscopy/transmission electron microscopy image analyses: Examples from the Barnett, Woodford, Haynesville, Marcellus, and Doig units. Aapg Bulletin 96 (2012) 1099-1119.

[4] L.J. Klinkenberg, The permeability of porous media to liquids and gases, Drilling and Production Practice, American Petroleum Institute, 1941, pp. 200-213.

[5] A. Beskok, and G.E. Karniadakis, A model for flows in channels, pipes, and ducts at micro and nano scales. Microscale Thermophysical Engineering 3 (1999) 43-77.

[6] F. Civan, Effective correlation of apparent gas permeability in tight porous media. Transport in Porous Media 82 (2010) 375-384.

[7] A. Mehmani, M. Prodanovic, and F. Javadpour, Multiscale, multiphysics network modeling of shale matrix gas flows. Transport in Porous Media 99 (2013) 377-390.

[8] J. Ma, J.P. Sanchez, K. Wu, G.D. Couples, and Z. Jiang, A pore network model for simulating non-ideal gas flow in micro-and nano-porous materials. Fuel 116 (2014) 498-508.

[9] R. Al-Hussainy, H. Ramey Jr, and P. Crawford, The flow of real gases through porous media. journal of Petroleum Technology 18 (1966) 624-636.

[10] M. Wang, and Z. Li, An Enskog based Monte Carlo method for high Knudsen number non-ideal gas flows. Computers \& Fluids 36 (2007) 1291-1297.

[11] M. Wang, X.D. Lan, and Z.X. Li, Analyses of gas flows in micro- and nanochannels. International Journal of Heat and Mass Transfer 51 (2008) 3630-3641.

[12] M. Wang, and Z.X. Li, Nonideal gas flow and heat transfer in micro- and nanochannels using the direct simulation Monte Carlo method. Physical Review E 68 (2003).

[13] M. Villazon, G. German, R.F. Sigal, F. Civan, and D. Devegowda, Parametric investigation of shale gas production considering nano-scale pore size distribution formation factor and non-Darcy flow mechanisms, SPE Annual Technical Conference and Exhibition, Society of Petroleum Engineers, 2011.

[14] H. Wang, and M. Marongiu-Porcu, Impact of Shale-Gas Apparent Permeability on Production: Combined Effects of Non-Darcy Flow/Gas-Slippage, Desorption, and Geomechanics. SPE Reservoir Evaluation \& Engineering (2015).

[15] S. Chen, and G.D. Doolen, Lattice Boltzmann method for fluid flows. Annual Review of Fluid Mechanics 30 (1998) 329-364.

[16] L. Chen, L. Zhang, Q. Kang, H.S. Viswanathan, J. Yao, and W. Tao, Nanoscale simulation of shale transport properties using the lattice Boltzmann method: permeability and diffusivity. Scientific Reports 5 (2015).

[17] S.Y. Chen, M.R. Wang, and Z.H. Xia, Multiscale fluid mechanics and modeling. Mechanics for the World: Proceedings of the 23rd International Congress of Theoretical and Applied Mechanics, Ictam2012 10 100-114.

[18] M.H.C. Knudsen, The kinetic theory of gases; some modern aspects, Methuen, London, 1933. 
[19] G.A. Bird, Definition of mean free path for real gases. Physics of Fluids 26 (1983) 3222-3223.

[20] J. Kozeny, Capillary motion of water in soils. Sitzungsberichte der Akademie der Wissenschaften in Wien, Mathematisch-Naturwissenschaftliche Klasse 136 (1927) 271-306.

[21] X.Y. He, and L.S. Luo, Theory of the lattice Boltzmann method: From the Boltzmann equation to the lattice Boltzmann equation. Physical Review E 56 (1997) 6811-6817.

[22] M. Wang, and S. Chen, Electroosmosis in homogeneously charged micro- and nanoscale random porous media. Journal of Colloid and Interface Science 314 (2007) 264-273.

[23] M. Wang, N. Pan, J.K. Wang, and S.Y. Chen, Lattice Poisson-Boltzmann simulations of electroosmotic flows in charged anisotropic porous media. Communications in Computational Physics 2 (2007) 1055-1070.

[24] M.R. Wang, and Q.J. Kang, Electrokinetic Transport in Microchannels with Random Roughness. Analytical Chemistry 81 (2009) 2953-2961.

[25] Y.H. Qian, D. Dhumieres, and P. Lallemand, Lattice BGK model for Navier-Stokes equation. Europhysics Letters 17 (1992) 479-484.

[26] J.K. Wang, M. Wang, and Z.X. Li, A lattice Boltzmann algorithm for fluid-solid conjugate heat transfer. International Journal of Thermal Sciences 46 (2007) 228-234.

[27] F.J. Higuera, S. Succi, and R. Benzi, Lattice gas-dynamics with enhanced collisions. Europhysics Letters 9 (1989) 345-349.

[28] P. Lallemand, and L.S. Luo, Theory of the lattice Boltzmann method: Dispersion, dissipation, isotropy, Galilean invariance, and stability. Physical Review E 61 (2000) 6546-6562.

[29] D. d'Humieres, I. Ginzburg, M. Krafczyk, P. Lallemand, and L.S. Luo, Multiple-relaxation-time lattice Boltzmann models in three dimensions. Philosophical Transactions of the Royal Society of London Series a-Mathematical Physical and Engineering Sciences 360 (2002) 437-451.

[30] C. Pan, L.-S. Luo, and C.T. Miller, An evaluation of lattice Boltzmann schemes for porous medium flow simulation. Computers \& Fluids 35 (2006) 898-909.

[31] S. Ansumali, and I.V. Karlin, Kinetic boundary conditions in the lattice Boltzmann method. Physical Review E 66 (2002).

[32] C. Cercignani, M. Lampis, and S. Lorenzani, Variational approach to gas flows in microchannels. Physics of Fluids 16 (2004) 3426-3437.

[33] K.K. Shah, and G. Thodos, A comparison of equation of state. Industrial and Engineering Chemistry 57 (1965) 30-\&.

[34] U. Setzmann, and W. Wagner, A new equation of state and tables of thermodynamic properties for methane covering the range from the melting line to $625 \mathrm{~K}$ at pressures up to $1000 \mathrm{MPa}$. Journal of Physical and Chemical Reference Data 20 (1991) 1061-1155.

[35] B.A. Younglove, and J.F. Ely, Thermophysical properties of fluids .2. methane, ethane, propane, isobutane, and normal butane. Journal of Physical and Chemical Reference Data 16 (1987) 577-798.

[36] A.S. Sangani, and A. Acrivos, Slow flow through a periodic array of spheres. International Journal of Multiphase Flow 8 (1982) 343-360.

[37] X.L. Wang, and M. Economides, Advanced natural gas engineering, Gulf Pub., 2009.

[38] M. Wang, J. Wang, N. Pan, and S. Chen, Mesoscopic predictions of the effective thermal conductivity for microscale random porous media. Physical Review E 75 (2007).

[39] M. Wang, and N. Pan, Predictions of effective physical properties of complex multiphase materials. Materials Science \& Engineering R-Reports 63 (2008) 1-30.

[40] M.J. Fetkovich, DECLINE CURVE ANALYSIS USING TYPE CURVES. Journal of Petroleum Technology 32 (1980) 1065-1077. 
[41] J. Arps, Analysis of decline curves. Transactions of the AIME 160 (1945) 228-247. 